\title{
Inhibition of Mitochondrial Complex I Aggravates Folic Acid-Induced Acute Kidney Injury
}

\author{
Wen Zhang ${ }^{a-d}$ Yunwen Yang ${ }^{a-c}$ Huiping Gao ${ }^{a-c}$ Yue Zhang ${ }^{a-c}$ \\ Zhanjun Jia ${ }^{a-c}$ Songming Huang ${ }^{a-c}$ \\ aDepartment of Nephrology, Children's Hospital of Nanjing Medical University, Nanjing, \\ PR China; ${ }^{b}$ Nanjing Key Laboratory of Pediatrics, Children's Hospital of Nanjing Medical \\ University, Nanjing, China; ' Jiangsu Key Laboratory of Pediatrics, Nanjing Medical \\ University, Nanjing, China; ${ }^{d}$ Department of Nephrology, Jiangsu Province Hospital on \\ Integration of Chinese and Western Medicine, Nanjing, China
}

\section{Keywords}

Rotenone · Folic acid · Mitochondrial dysfunction · Acute kidney injury · Oxidative stress

\begin{abstract}
Background: Some researches revealed that mitochondrial dysfunction is associated with various kidney injury. However, the role of mitochondrial dysfunction in the pathogenesis of acute kidney injury (AKI) still needs evidence. Methods: We evaluated the effect of mitochondrial complex I inhibitor rotenone on folic acid (FA)-induced AKI in mice. Results: Strikingly, the mice pretreated with rotenone at a dose of $200 \mathrm{ppm}$ in food showed exacerbated kidney injury as shown by higher levels of blood urea nitrogen and creatinine compared with FA alone group. Meanwhile, both renal tubular injury score and the expression of renal tubular injury marker neutrophil gelatinase-associated lipocalin were further elevated in rotenone-pretreated mice, suggesting the deteriorated renal tubular injury. Moreover, the decrements of mitochondrial DNA copy number and the expressions of mitochondrial Cytochrome c oxidase subunit 1, mitochondrial NADH dehydrogenase subunit 1, and mitochondria-specific superoxide dismutase (SOD2) in the kidneys of FA-treated mice were further reduced in rotenonepretreated mice, indicating the aggravated mitochondrial damage. In parallel with the SOD2 reduction, the oxidative stress markers of malondialdehyde and $\mathrm{HO}-1$ displayed greater increment in AKI mice with rotenone pretreatment in line with the deteriorated apoptotic response
\end{abstract}

W.Z. and Y.Y. contributed equally to this work. 


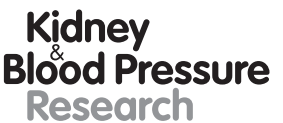

Research

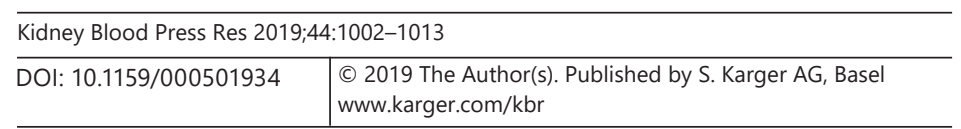

Zhang et al.: Inhibition of Mitochondrial Complex I Aggravates FA-Induced AKI

and inflammation. Conclusion: Our results suggested that the inhibition of mitochondrial complex I activity aggravated renal tubular injury, mitochondrial damage, oxidative stress, cell apoptosis, and inflammation in FA-induced AKI.

(C) 2019 The Author(s)

Published by S. Karger AG, Basel

\section{Introduction}

Acute kidney injury (AKI) has been estimated with a mortality rate of $\sim 50 \%[1,2]$. It can arise in a variety of pathological conditions such as exposure to nephrotoxins and ischemia $[3,4]$. After injury, inflammation, oxidative stress, and apoptosis are common pathological features of AKI, which may eventually lead to tubulointerstitial fibrosis and chronic kidney disease (CKD) [5]. Patients with AKI have a higher risk in the development of CKD and endstage renal disease [6]. Currently, there are no satisfactory therapies for AKI.

Recently, some researches revealed an abnormal change of mitochondria in CKD models of UUO [7-9] and 5/6 nephrectomy [10]. Mitochondrial damage enhanced the production of reactive oxygen species (ROS) and the release of proapoptotic factors, resulting in the oxidative stress, cellular apoptosis, and inflammation $[11,12]$.

Rotenone could inhibit the activity of mitochondrial respiratory chain complex I via affecting the transfer of the iron-sulfur clusters and coenzyme $Q$ to reduce the synthesis of ATP [13]. By reviewing the literatures, there exist inconsistent results about the effect of rotenone on kidney diseases. Sun and Zhang et al. $[14,15]$ reported that rotenone attenuates oxidative stress, inflammation, and fibrosis in CKD models of UUO and ischemia-reperfusioninduced CKD. Oppositely, Jiang et al. [16] demonstrated that rotenone induces nephrotoxicity in rats in line with the enhanced oxidative stress and apoptosis. So far, the role of mitochondrial inhibitors in AKI has not been well studied, and the role of mitochondrial inhibitor in different AKI models still needs to be defined. Folic acid (FA) overdose-induced AKI has been described in humans [17] and recapitulates the major processes of human AKI. Thus, FA-induced AKI animal model was widely used to investigate the pathogenesis and therapies of AKI. In the present study, we tested the role of mitochondrial complex I inhibitor rotenone in FA-induced AKI and the underlying mechanisms in mice.

\section{Materials and Methods}

In some experiments, we followed the methods from Zhang et al. [15].

\section{Reagents and Antibodies}

Rotenone (R8875) and FA (F7876) were purchased from Sigma (St. Louis, MO, USA). Antibodies against neutrophil gelatinase-associated lipocalin (NGAL; ab63929) were purchased from Abcam (Shanghai, China). BAX (50599-2-Ig, 1:1,000), HO-1 (10701-1-AP, 1:1,000), superoxide dismutase (SOD2; 24127-1-AP, 1:1,000), GAPDH (10494-1-AP, 1:1,000), and $\beta$-actin (20536-1-AP, 1:1,000) were purchased from Proteintech (Wuhan, China). Horseradish peroxidase-conjugated goat antirabbit secondary antibody was purchased from Beyotime (Haimen, china, A0208, 1:1,000). SYBR Green master mix for real-time polymerase chain reaction (RT-PCR) was provided by Applied Biosystems (4367660, Foster City, CA, USA).

\section{Animals}

C57BL/6J mice were originally obtained from Jackson lab. This mouse colony was propagated at the Nanjing Medical University. Male mice with the age at 8-12 weeks old were used 


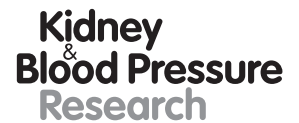

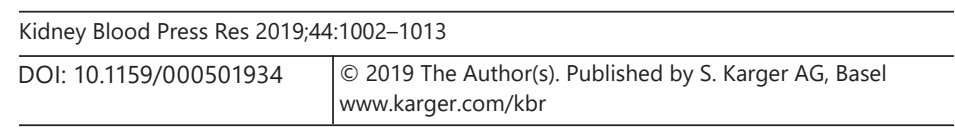

Zhang et al.: Inhibition of Mitochondrial Complex I Aggravates FA-Induced AKI

for experiments. All mice were maintained on a $12 \mathrm{~h}$ light-dark cycle in a temperaturecontrolled $\left(22 \pm 2{ }^{\circ} \mathrm{C}\right)$ room, were fed a standard rodent diet, and were allowed to free access to drinking water. The procedures were approved by the Nanjing Medical University Institutional Animal Care and Use Committees.

\section{FA-Induced AKI Model and Rotenone Treatment}

Mice were injected (i.p.) with FA at $240 \mathrm{mg} / \mathrm{kg}$ body weight. Sodium bicarbonate $(0.3 \mathrm{M}$ $\mathrm{NaHCO}_{3}$, the vehicle used for FA administration) alone was used as controls. To examine the effect of the inhibition of the mitochondrial complex I on FA-induced AKI, mice were pretreated with rotenone at $200 \mathrm{ppm}$ in jelly diet for $24 \mathrm{~h}$ before FA i.p. injection. The dose of rotenone at $200 \mathrm{ppm}$ in jelly diet used in this study was according to our previous studies [14, 15, 18-20]. The sham control mice were treated with jelly diet without rotenone. After FA administration for $72 \mathrm{~h}$, the animals were killed and the kidney tissues were harvested for the evaluation of gene and protein expressions and histology. Six mice were used in each group.

\section{Measurements of Blood Urea Nitrogen and Creatinine}

Blood urea nitrogen (BUN) and creatinine levels were determined to assess the renal function. After the FA treatment for $72 \mathrm{~h}$, the mice were sacrificed, and the blood samples were obtained from the inferior vena cave and anticoagulated with heparin $(20 \mathrm{unit} / \mathrm{mL}$ blood). The plasma was isolated by centrifugation. Automatic biochemical analyzer was used to detect the concentration of BUN and creatinine.

\section{Renal Histology and Tubular Injury Score}

Kidney tissues from mice were fixed with $4 \%$ paraformaldehyde, embedded in paraffin, and sectioned transversely. Paraffin sections $(3 \mu \mathrm{m})$ of renal tissue were stained with periodic acid-Schiff and examined by light microscopy. Ten nonoverlapping tubulointerstitial fields were randomly selected from each slide. The swelling of renal tubular epithelial cells, renal tubular atrophy, lumen dilatation, and brush edge shedding were observed under 200x magnification by microscopy. According to the percentage of pathological injury area under a single field of vision, semi-quantitative scoring was performed by 4 points. The scoring criteria are as follows as described previously $[21,22]$ : the injury area is $0 \%, 0$ points, normal; injury area $<25 \%, 1$ point, mild; injury area is $25-50 \%, 2$ points, moderate; injury area is $50-75 \%, 3$ points, severe; injury area $>75 \%, 4$ points, extremely severe.

\section{Western Blotting}

Kidney tissues $(20 \mathrm{mg})$ were lysed used RIPA lysis $(200 \mu \mathrm{L})$, which contained protease inhibitors. Immunoblotting was then performed using primary antibodies against NGAL (1: 1,000), HO-1 (1:500), SOD2 (1:1,000), BAX (1:500), $\beta$-actin $(1: 1,000)$, and GAPDH $(1: 1,000)$, followed by addition of HRP-labeled secondary antibodies. The blots were visualized using Amersham ECL Detection Systems (Amersham, Buckinghamshire, UK). The intensity of the band was quantitatively analyzed using the gel imaging system scan (Bio-rad, USA).

\section{Quantitative RT-PCR}

Total DNA and RNA from kidney cortex tissues were isolated using the DNeasy Tissue Kit (Tiangen, China) and Trizol reagent (Takara), respectively. Primers were designed using Primer5 software (available at http://frodo.wi.mit.edu/) and synthesized by Invitrogen. The sequences of primers for quantitative RT-PCR were shown in Table 1. Reverse transcription was done by using Promega Reverse Transcription System according to the manufacturer's instruction (Madison, WI, USA). RT-PCR amplification was performed using the ABI 7500 RT-PCR Detection System (Foster City, CA, USA) and SYBR Premix Ex Taq (TaKaRa). The 
Table 1. Sequences of primers for qRT-PCR

\begin{tabular}{|c|c|}
\hline Gene & Primer sequence \\
\hline GAPDH & $\begin{array}{l}\text { 5'-GTCTTCACTACCATGGAGAAGG-3' } \\
\text { 5'-TCATGGATGACCTTGGCCAG-3' }\end{array}$ \\
\hline NGAL & $\begin{array}{l}\text { 5'-GCAGGTGGTACGTTGTGGG-3' } \\
\text { 5'-CTCTTGTAGCTCATAGATGGTGC-3' }\end{array}$ \\
\hline IL-6 & $\begin{array}{l}\text { 5'-GCTTAGGCATAACGCACT-3' } \\
\text { 5'-GGAAATCGTGGAAATGAG-3' }\end{array}$ \\
\hline ICAM-1 & $\begin{array}{l}\text { 5'-CGCTTCCGCTACCATCAC-3' } \\
\text { 5'-GGCGGCTCAGTATCTCCTC-3' }\end{array}$ \\
\hline mt-COX1 & $\begin{array}{l}\text { 5'-CCCAATCTCTACCAGCATC-3' } \\
\text { 5'-GGCTCATAGTATAGCTGGAG-3' }\end{array}$ \\
\hline mt-ND1 & $\begin{array}{l}\text { 5'-AATCGCCATAGCCTTCCTAACAT-3' } \\
\text { 5'-GGCGTCTGCAAATGGTTGTAA-3' }\end{array}$ \\
\hline 18s rRNA & $\begin{array}{l}\text { 5'-TTCGGAACTGAGGCCATGATT-3' } \\
\text { 5'-TTTCGCTCTGGTCCGTCTTG-3' }\end{array}$ \\
\hline \multicolumn{2}{|c|}{$\begin{array}{l}\text { qRT-PCR, quantitative real-time-PCR; IL-6, interleukin-6; mt-COX1, } \\
\text { mitochondrial Cytochrome c oxidase subunit 1; mt-ND1, mitochondri- } \\
\text { al NADH dehydrogenase subunit } 1 \text {; NGAL, neutrophil gelatinase- } \\
\text { associated lipocalin. }\end{array}$} \\
\hline
\end{tabular}

cycling program includes a preliminary denaturation $\left(95^{\circ} \mathrm{C}\right.$ for $\left.10 \mathrm{~min}\right)$ followed by 40 cycles $\left(95^{\circ} \mathrm{C}\right.$ for $15 \mathrm{~s}$ and $60^{\circ} \mathrm{C}$ for $\left.1 \mathrm{~min}\right)$. The relative mitochondrial DNA copy number was normalized to the 18S rRNA level encoded by the nuclear DNA, and mRNA levels were normalized to GAPDH and calculated using the comparative cycle threshold ( $\Delta \Delta \mathrm{Ct}$ ) method.

\section{Malondialdehyde Determination}

We used a commercial kit toquantify the generation of malondialdehyde (MDA) according to the manufacturer's protocol. In brief, kidney tissue samples were homogenized and sonicated in RIPA buffer on ice. Tissue lysates were then centrifuged at $12,000 \mathrm{~g}$ for $15 \mathrm{~min}$ at $4{ }^{\circ} \mathrm{C}$ to collect the supernatant. Renal MDA was measured using a Lipid Peroxidation MDA assay kit (Beyotime). MDA levels were detected using multimode microplate readers (SpectramMax M5) at $532 \mathrm{~nm}$ and normalized by the protein concentration.

In situ Transferase dUTP Nick End Labeling Assays

For terminal deoxynucleotidyl transferase dUTP nick end labeling (TUNEL) assay, kidney sections were stained as per the manufacturer's instructions (TUNEL Detection Kit, 11684817910, Roche). The number of TUNEL-positive nuclei per field was evaluated in 5 fields per section and 5 sections per kidney.

\section{Enzyme-Linked Immunosorbent Assay}

The kidney tissue was homogenized in phosphate-buffered saline and then centrifuged for $5 \mathrm{~min}$ at 10,000 rpm. Supernatant was diluted at 1:50 with enzyme immunoassay buffer. The plasma tumor necrosis factor- $\alpha$ (TNF- $\alpha$ ) level was determined by an enzyme-linked immunosorbent assay kit (DKW12-2720, Dakewe Bioengineering Co., Ltd., China) according to the manufacturer's instructions. 


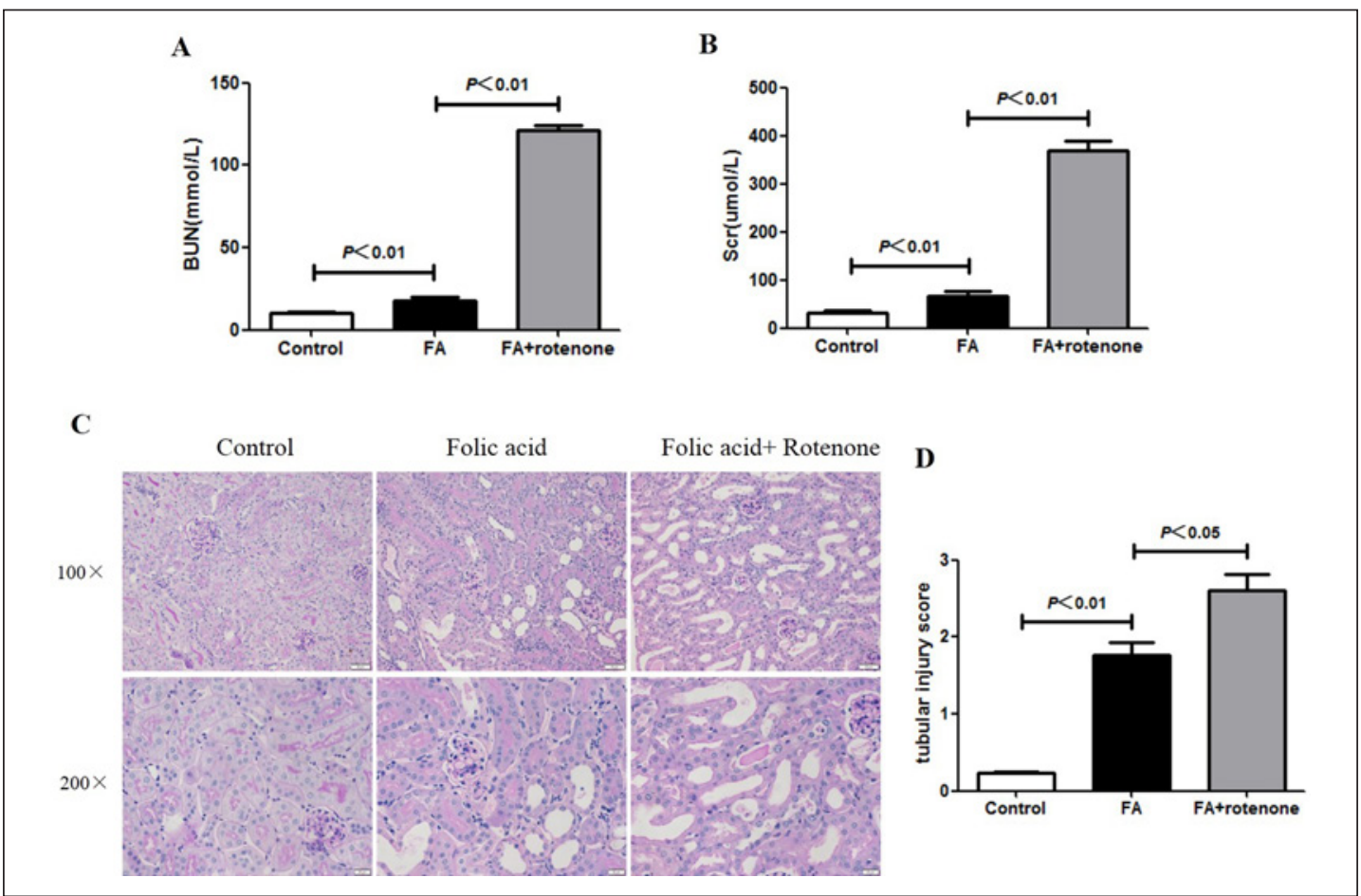

Fig. 1. Rotenone exacerbated FA-induced renal injury. A BUN. B Blood serum creatinine. C Representative images of renal PAS staining. D Tubular injury score. Data were presented as means \pm SE, $n=6$ in each group. BUN, blood urine nitrogen; FA, folic acid; Scr, serum creatinine.

\section{Statistical Analysis}

The quantitative data of the experiment were expressed by means \pm SEM. Statistical analysis was performed using SPSS 16.0 software. Statistical significance was determined by Student $t$ test for comparisons of 2 groups. Multigroup comparisons were performed using ANOVA multiple comparisons. $p<0.05$ was considered statistically significant.

\section{Results}

\section{Rotenone Exacerbated FA-Induced Renal Injury}

To evaluate the effect of rotenone on renal function in FA-treated mice, we measured BUN and creatinine levels. Compared with the FA alone group, rotenone treatment further increased BUN (from $18.08 \pm 0.76$ to $121.02 \pm 1.08 \mathrm{mM}$ ) and serum creatinine (from $67.40 \pm$ 3.45 to $368.13 \pm 7.91 \mu \mathrm{M}$ ) levels in AKI mice (Fig. 1A, B). In line with the renal dysfunction, periodic acid-Schiff staining of kidney tissues showed that FA-induced renal tubular injury was further aggravated by rotenone pretreatment as shown by the more sever tubular dilation, protein cast formation, and brush border loss (Fig. 1C, D). Furthermore, the tubular injury marker NGAL was measured to better evaluate the renal tubular injury. A shown by the data, both protein and mRNA levels were significantly upregulated by FA treatment, which was further elevated by rotenone pretreatment (Fig. 2A-C). These data suggested that inhibition of mitochondrial complex I activity by rotenone aggravated acute renal tubular injury induced by FA. 
Fig. 2. Effect of rotenone pretreatment on the regulation of tubular injury marker NGAL induced by FA. A Western blotting analysis of NGAL. GAPDH was used as loading control. B Densitometry of Western blots in A. C qRT-PCR analysis of NGAL. Data were presented as means $\pm \mathrm{SE}, n=$ 6 in each group. FA, folic acid; NGAL, neutrophil gelatinase-associated lipocalin.

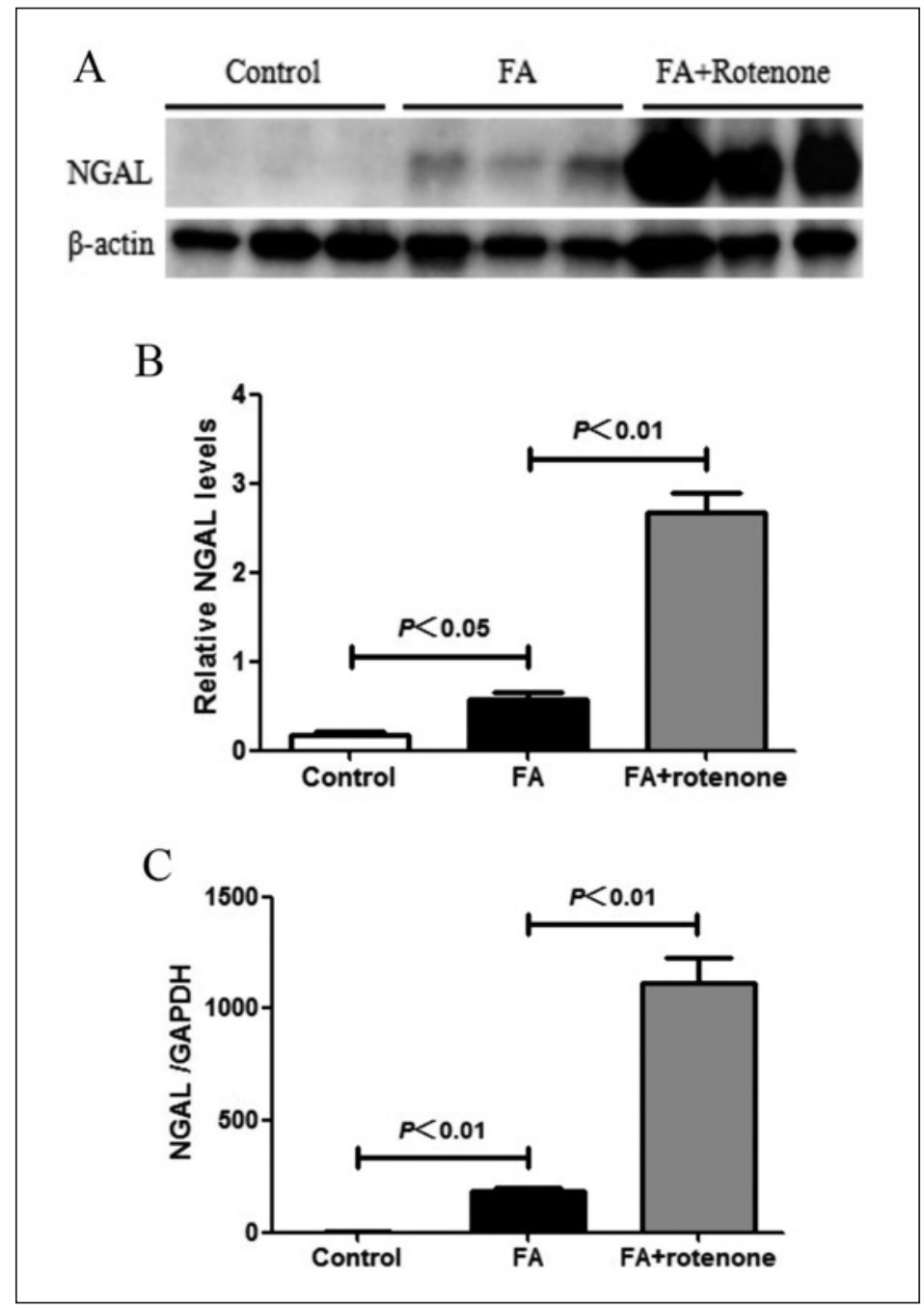

Rotenone Treatment Deteriorated Renal Mitochondrial Damage Induced by FA

Mitochondrial dysfunction has been considered as one of the pathogenic factors in kidney injury [23]. Thus, we measured mtDNA copy number and mitochondrial genes of mitochondrial Cytochrome c oxidase subunit 1 (mt-COX1) and mitochondrial NADH dehydrogenase subunit 1 (mt-ND1) to evaluate the mitochondrial abnormality. As shown by Figure 3A-C, in FA-treated mice, the mtDNA copy number and the expressions of mt-COX1 and mt-ND1 were obviously reduced, which was further worsened in rotenone-treated AKI mice, suggesting deterioration of mitochondrial abnormality. Furthermore, we also measured mitochondriaspecific SOD2 and found that rotenone treatment led to more severe reduction of SOD2 levels in the kidney of mice challenged with FA (Fig. 3D, E), suggesting the impairment of the antioxidant system in mitochondria.

\section{Rotenone Aggravated Renal Oxidative Stress Induced by FA}

Dysfunctional mitochondria serve as the important source of ROS production. Contrasting to the reduced SOD2 expression in rotenone-treated AKI mice, the levels of kidney MDA were further enhanced compared to FA alone group (Fig. 4A). Similarly, another oxidative stress marker HO-1 was markedly higher in the kidneys of mice with rotenone pretreatment than 

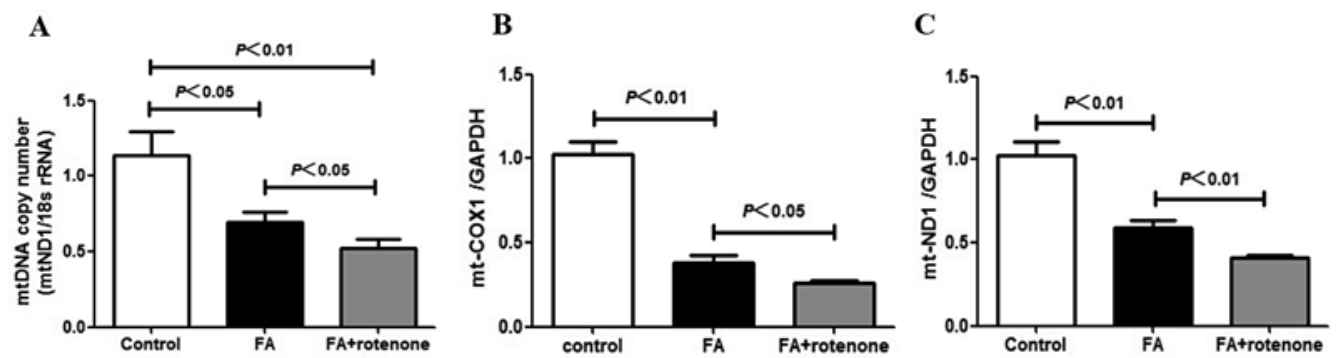

D

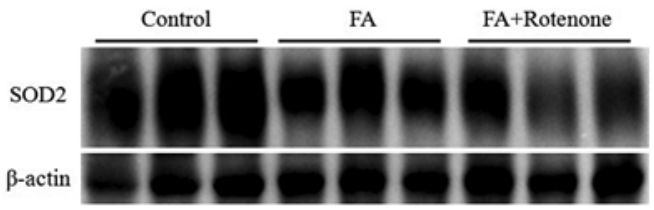

$\mathbf{E}$

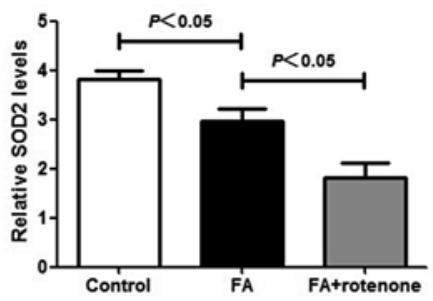

Fig. 3. Rotenone pretreatment worsened renal mitochondrial damage induced by FA. A mtDNA copy number was determined by qRT-PCR. B qRT-PCR analysis of mt-COX1. C qRT-PCR analysis of mt-ND1. D Western blotting analysis of SOD2, GAPDH was used as loading control. E Densitometry of Western blots in D. Data were presented as means $\pm \mathrm{SE}, n=6$ in each group. FA, folic acid; mt-COX1, mitochondrial Cytochrome c oxidase subunit 1; SOD2, superoxide dismutase; mt-ND1, mitochondrial NADH dehydrogenase subunit 1.

that in FA alone group (Fig. 3C). These findings indicated that rotenone treatment promoted renal oxidative stress in FA-induced AKI mice possibly through the impairment of mitochondrial function.

\section{Rotenone Aggravated Renal Cellular Apoptosis Induced by FA}

Cellular apoptosis is a common feature of many forms of AKI. By TUNEL staining, we found that the TUNEL-positive nuclei were almost undetectable in the control kidneys, while the number of TUNEL positive nuclei was significantly increased after FA insult. In contrast, rotenone pretreatment evidently increased the number of TUNEL-positive nuclei compared to the FA alone group (Fig. 5A, B). To better evaluate the apoptotic response, we further measured BAX expression in the kidneys and found that FA-induced upregulation of BAX protein was more robustly increased in rotenone-pretreated animals (Fig. 5C, D). These data suggested that rotenone could aggravate apoptotic response in $\mathrm{FA}$-induced AKI.

\section{Rotenone Promoted the Renal Inflammation Induced by FA}

It is well known that the inflammation is involved in the pathogenesis of AKI [24]. By quantitative RT-PCR, we found that the inflammatory markers interleukin-6 (IL-6) were markedly elevated in FA-treated mice, which was further upregulated in rotenone-pretreated mice (Fig. 6A). Furthermore, we measured circulating levels of TNF- $\alpha$ by enzyme-linked immunosorbent assay and observed a trend increment in rotenone-pretreated mice compared to FA alone group (Fig. 6C). However, the upregulation of ICAM-1 mRNA in kidneys induced by FA was unaltered by rotenone (Fig. 6B). These data suggested that rotenone promoted the inflammatory response in FA-induced AKI. 
Fig. 4. Rotenone aggravated renal oxidative stress induced by FA. A Determination of renal tissue MDA. B Western blotting analysis of HO-1, $\beta$-actin was used as loading control. C Densitometry of Western blots in B. Data were presented as means $\pm \mathrm{SE}, n=6$ in each group. FA, folic acid; MDA, malondialdehyde.
$\mathbf{A}$

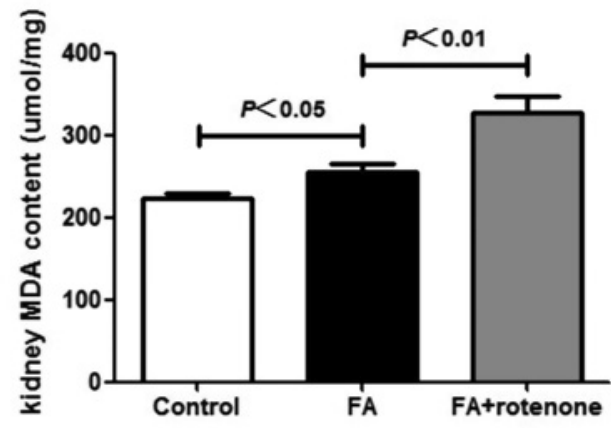

B

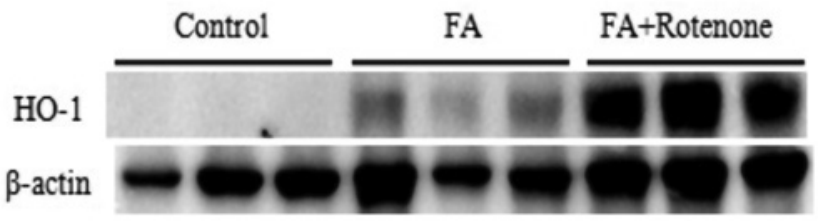

C

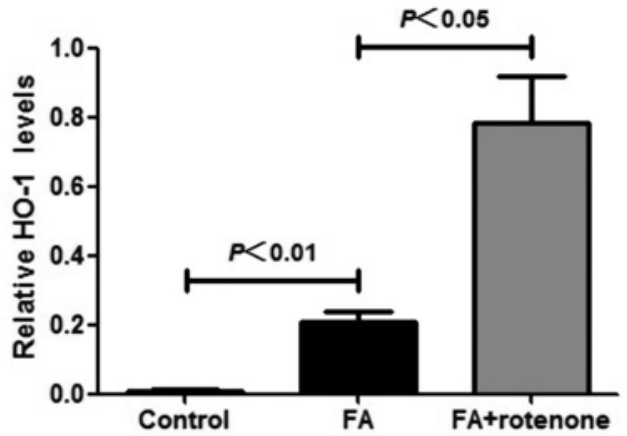

\section{Evaluation of the Rotenone Toxicity in Mice}

Finally, we assessed the toxicity of rotenone in mice. As shown by the data, 4-day rotenone administration did not significantly affect the body weight, and the circulation levels of alanine aminotransferase (ALT), aspartate aminotransferase (AST), lactate dehydrogenase (LDH), BUN, and Cr (Fig. 7A-F). These data suggested rotenone at a dose of $200 \mathrm{ppm}$ in food did not cause the obvious organ injury under the normal condition.

\section{Discussion}

In a number of experimental studies, rotenone is used to induce the Parkinson's disease in animals [25-27]. Studies in cancer research indicated that rotenone could be effective in treating cancers including liver and breast cancer [28, 29]. Recent studies from our group reported that rotenone treatment protected the kidneys from obstructive injury and ischemiareperfusion-induced CKD, possibly via inhibiting the activity of the damaged mitochondria $[14,15]$. In ischemia-reperfusion-induced CKD, we applied the rotenone to the animals after acute injury, which significantly attenuated the progression of AKI to CKD. In addition, another 


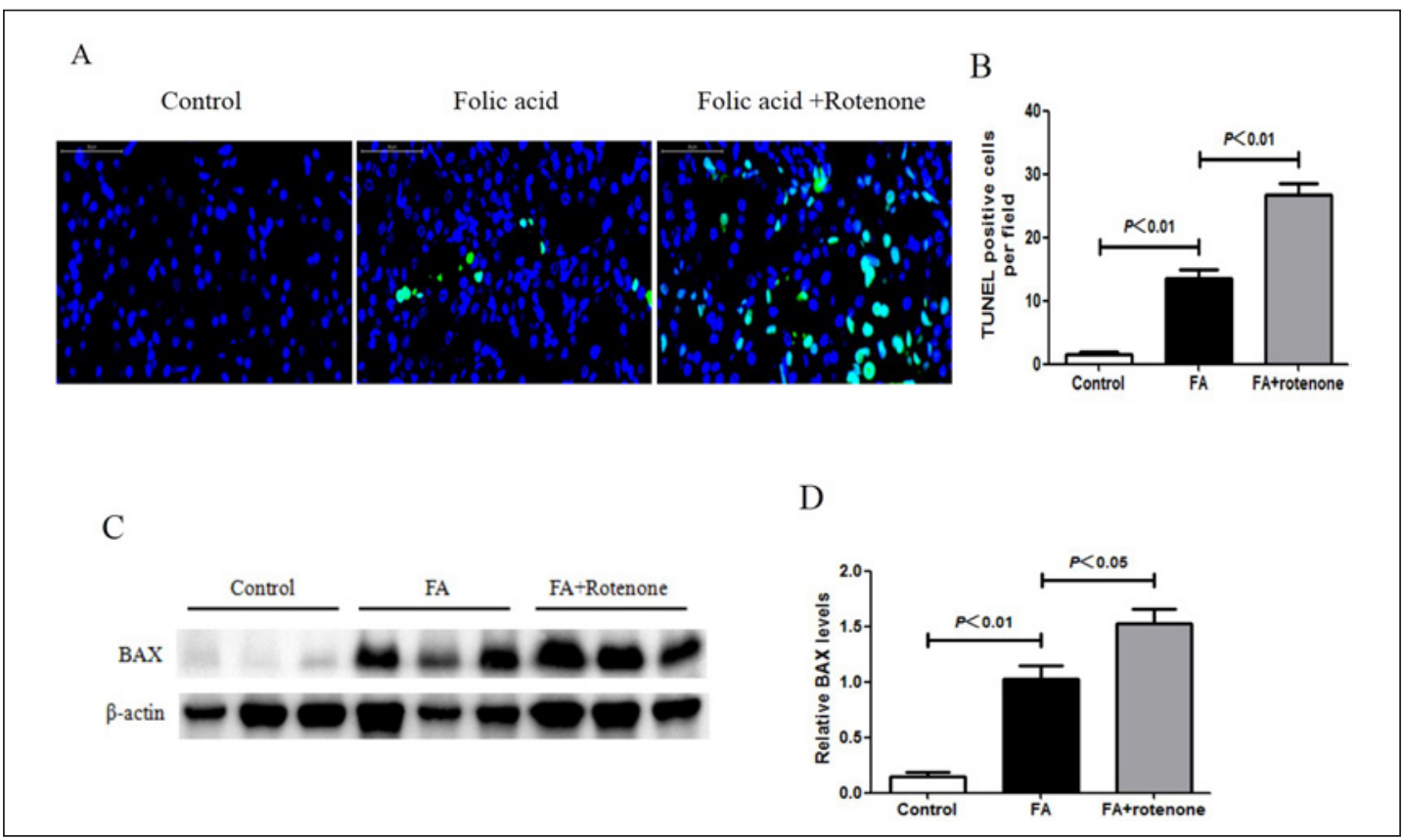

Fig. 5. Rotenone aggravated renal cellular apoptosis induced by FA. A Representative images of renal TUNEL staining (green). B Quantification of TUNEL positive cell in A. C Western blotting analysis of BAX, GAPDH was used as loading control. D Densitometry of Western blots in C. Data were presented as means \pm SE, $n=6$ in each group. FA, folic acid; TUNEL, transferase dUTP nick end labeling.

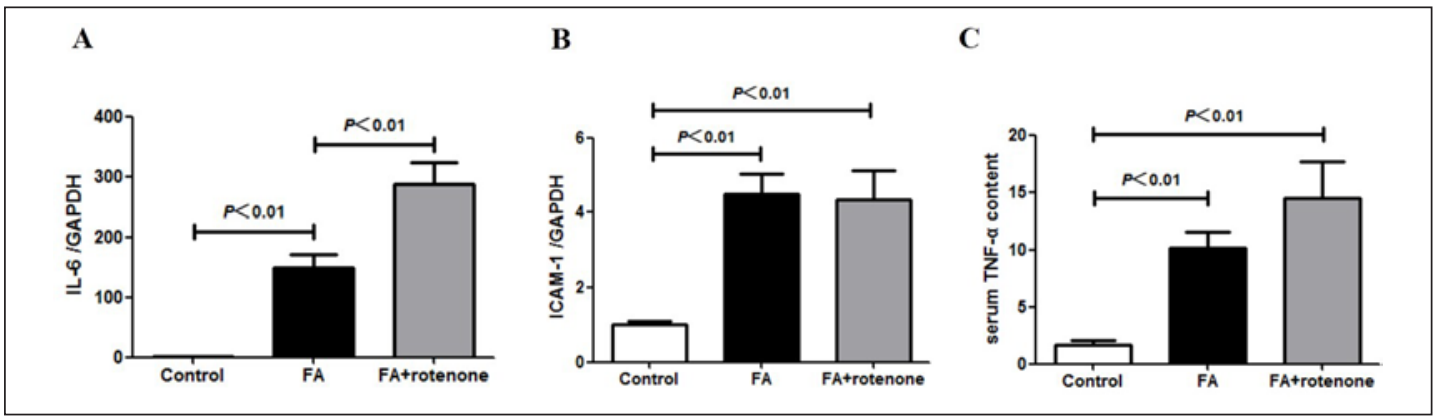

Fig. 6. Rotenone promoted renal inflammation induced by FA. A, B The mRNA expressions of IL-6 and ICAM1 in renal tissues were detected by qRT-PCR. C Enzyme-linked immunosorbent assay analysis of renal TNF- $\alpha$ protein level. Data were presented as means \pm SE, $n=6$ in each group. FA, folic acid; IL-6, interleukin-6; TNF- $\alpha$, tumor necrosis factor- $\alpha$.

study found that rotenone could ameliorate aldosterone-induced renal injury [30]. However, the role of rotenone in AKI has not been studied. In the present study, our results showed that rotenone further exacerbated FA-induced renal injury possibly by aggravating mitochondrial dysfunction, oxidative stress, apoptosis, and inflammation in the kidney.

Mitochondrial dysfunction has been recognized as a pathogenic feature in FA-induced kidney injury [31]. Our results displayed FA treatment significantly decreased mtDNA copy number and the expressions of mitochondrial gene mt-COX1 and mt-ND1, while rotenone pretreatment makes the situation worse, suggesting the deterioration of mitochondrial abnormality. Mitochondrial damage not only results in the reduction of ATP level but also leads to the excessive production of ROS, which directly causes cellular injury and subsequently promotes 


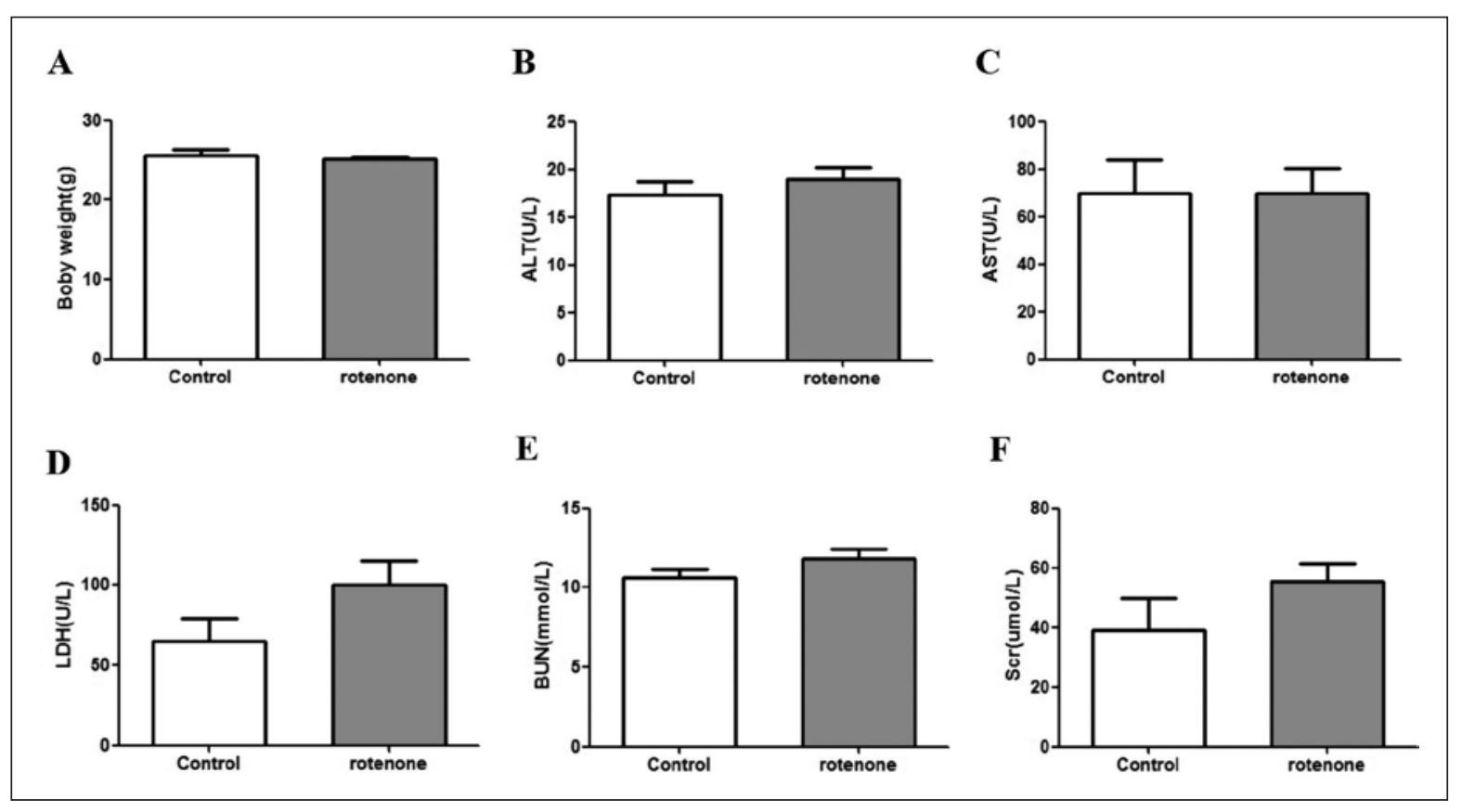

Fig. 7. Evaluation of rotenone toxicity in mice. A Body weight after 4-day rotenone treatment. B-F Blood levels of aspartate aminotransferase (B), alanine aminotransferase (C), LDH (D), BUN (E), and serum creatinine (F). Data were presented as means \pm SE, $n=6$ in each group. Scr, serum creatinine; BUN, blood urea nitrogen; ALT, alanine aminotransferase; AST, aspartate aminotransferase.

mitochondrial dysfunction [32,33]. It is well accepted that oxidative stress is involved in the pathogenesis of FA-induced kidney injury. Previous study has shown that rotenone could decrease the activity of antioxidant enzymes in the body [34], leading to the accumulation of a large number of free radicals, which causes oxidative damage [34]. Several groups have reported that rotenone administration may result in massive production of ROS [35, 36]. Verma et al. [37] reported that the MDA levels were significantly enhanced after rotenone treatment in animals. In agreement with previous studies mentioned above, in the present study, rotenone administration further decreased SOD2 level in renal tissue companied with increased kidney MDA content and HO-1 expression, indicating that rotenone might enhance the oxidative stress response via decreasing the activity antioxidant enzyme SOD2 in mitochondria.

Oxidative stress and other detrimental factors adversely affect mitochondrial function and leading to the cell apoptosis. Previous findings have shown that apoptosis acts as another important mechanism in FA-induced AKI [38]. As shown by the results, significant apoptosis was observed in kidneys after treatment with FA as illustrated by TUNEL staining and BAX measurement, while rotenone pretreatment aggravated FA-induced apoptotic response. Rotenone-induced oxidative stress has been shown to cause cell apoptosis [39, 40]. In turn, oxidative stress could lead to more severe mitochondrial damage and cell apoptosis [34, 41].

Inflammation plays an important role in the occurrence and development of AKI [24]. In the present study, FA treatment significantly upregulated the expressions of IL-6, TNF- $\alpha$, and ICAM-1. While, rotenone pretreatment further upregulated the IL- 6 expression. IL- 6 is produced mainly by activated macrophages [42] and exerts its effects by recruiting neutrophils to the peritubular region [43]. Also, it has been demonstrated that oxidative stress could trigger the inflammatory response in many pathological processes [44]. Thus, this enhanced inflammatory response could be trigged by the mitochondrial oxidative stress caused by mitochondrial complex I inhibitor rotenone. 


\section{Conclusion}

In summary, this study examined the role of mitochondrial complex I inhibitor rotenone in FA-induced AKI for the first time. The findings demonstrated that the inhibition of mitochondrial activity could be pathogenic in FA-induced AKI, which offered direct evidence showing the role of mitochondrial dysfunction in promoting AKI.

\section{Disclosure Statement}

The authors declare that there are no conflicts of interest.

\section{Funding Source}

This work was supported by Grants from the National Key Research and Development Program (no. 2016YFC0906103) and the National Natural Science Foundation of China (nos. 81600557, 81600532, 81600352, 81370802, 81300591, 81670647, and 81570616).

\section{Data Availability Statement}

All the data used to support the findings of this study are included within the article.

\section{References}

1 Chuasuwan A, Kellum JA. Acute kidney injury and its management. Contrib Nephrol. 2011;171:218-25.

2 Hoste EA, Schurgers M. Epidemiology of acute kidney injury: how big is the problem? Crit Care Med. 2008 Apr; 36(4 Suppl):S146-51.

3 Kinsey GR, Okusa MD. Pathogenesis of acute kidney injury: foundation for clinical practice. Am J Kidney Dis. 2011 Aug;58(2):291-301.

4 Yang L, Humphreys BD, Bonventre JV. Pathophysiology of acute kidney injury to chronic kidney disease: maladaptive repair. Contrib Nephrol. 2011;174:149-55.

5 Bellomo R, Kellum JA, Ronco C. Acute kidney injury. Lancet. 2012 Aug;380(9843):756-66.

6 Siew ED, Parr SK, Abdel-Kader K, Eden SK, Peterson JF, Bansal N, et al. Predictors of Recurrent AKI. J Am Soc Nephrol. 2016 Apr;27(4):1190-200.

7 García IM, Altamirano L, Mazzei L, Fornés M, Molina MN, Ferder L, et al. Role of mitochondria in paricalcitolmediated cytoprotection during obstructive nephropathy. Am J Physiol Renal Physiol. 2012 Jun; 302(12):F1595-605.

8 Small DM, Coombes JS, Bennett N, Johnson DW, Gobe GC. Oxidative stress, anti-oxidant therapies and chronic kidney disease. Nephrology (Carlton). 2012 May;17(4):311-21.

9 Xu Y, Ruan S, Wu X, Chen H, Zheng K, Fu B. Autophagy and apoptosis in tubular cells following unilateral ureteral obstruction are associated with mitochondrial oxidative stress. Int J Mol Med. 2013 Mar;31(3):62836.

10 Chen JF, Liu H, Ni HF, Lv LL, Zhang MH, Zhang AH, et al. Improved mitochondrial function underlies the protective effect of pirfenidone against tubulointerstitial fibrosis in 5/6 nephrectomized rats. PLoS One. 2013 Dec;8(12):e83593.

11 Heid ME, Keyel PA, Kamga C, Shiva S, Watkins SC, Salter RD. Mitochondrial reactive oxygen species induces NLRP3-dependent lysosomal damage and inflammasome activation. J Immunol. 2013 Nov;191(10):5230-8.

12 Michel S, Wanet A, De Pauw A, Rommelaere G, Arnould T, Renard P. Crosstalk between mitochondrial (dys) function and mitochondrial abundance. J Cell Physiol. 2012 Jun;227(6):2297-310.

13 Hsiao YP, Huang HL, Lai WW, Chung JG, Yang JH. Antiproliferative effects of lactic acid via the induction of apoptosis and cell cycle arrest in a human keratinocyte cell line (HaCaT). J Dermatol Sci. 2009 Jun;54(3):17584.

14 Sun Y, Zhang Y, Zhao D, Ding G, Huang S, Zhang A, et al. Rotenone remarkably attenuates oxidative stress, inflammation, and fibrosis in chronic obstructive uropathy. Mediators Inflamm. 2014;2014:670106. 
Zhang et al.: Inhibition of Mitochondrial Complex I Aggravates FA-Induced AKI

15 Zhang W, Sha Y, Wei K, Wu C, Ding D, Yang Y, et al. Rotenone ameliorates chronic renal injury caused by acute ischemia/reperfusion. Oncotarget. 2018 May; 9(36):24199-208.

16 Jiang XW, Qiao L, Feng XX, Liu L, Wei QW, Wang XW, et al. Rotenone induces nephrotoxicity in rats: oxidative damage and apoptosis. Toxicol Mech Methods. 2017 Sep;27(7):528-36.

17 Metz-Kurschel U, Kurschel E, Wagner K, Aulbert E, Graben N, Philipp T. Folate nephropathy occurring during cytotoxic chemotherapy with high-dose folinic acid and 5-fluorouracil. Ren Fail. 1990;12(2):93-7.

18 Yang Y, Fu Y, Wang P, Liu S, Sha Y, Zhang Y, et al. Intervention of mitochondrial activity attenuates cisplatininduced acute kidney injury. Int Urol Nephrol. 2019 Jul;51(7):1207-18.

19 Hua H, Ge X, Wu M, Zhu C, Chen L, Yang G, et al. Rotenone Protects Against Acetaminophen-Induced Kidney Injury by Attenuating Oxidative Stress and Inflammation. Kidney Blood Press Res. 2018;43(4):1297-309.

20 Sun Y, Zhang Y, Zhu Y, Zhang A, Huang S, Yin X, et al. Inhibition of mitochondrial complex-1 restores the downregulation of aquaporins in obstructive nephropathy. Am J Physiol Renal Physiol. 2016 Oct;311(4):F777-86.

21 Weidemann A, Bernhardt WM, Klanke B, Daniel C, Buchholz B, Câmpean V, et al. HIF activation protects from acute kidney injury. J Am Soc Nephrol. 2008 Mar;19(3):486-94.

22 Fang TC, Alison MR, Cook HT, Jeffery R, Wright NA, Poulsom R. Proliferation of bone marrow-derived cells contributes to regeneration after folic acid-induced acute tubular injury. J Am Soc Nephrol. 2005 Jun;16(6): 1723-32.

23 Ishimoto Y, Inagi R. Mitochondria: a therapeutic target in acute kidney injury. Nephrol Dial Transplant. 2016 Jul;31(7):1062-9.

24 Kinsey GR, Li L, Okusa MD. Inflammation in acute kidney injury. Nephron, Exp Nephrol. 2008;109(4):e102-7.

25 Liu Y, Sun JD, Song LK, Li J, Chu SF, Yuan YH, et al. Environment-contact administration of rotenone: A new rodent model of Parkinson's disease. Behav Brain Res. 2015 Nov;294:149-61.

26 Tanner CM, Kamel F, Ross GW, Hoppin JA, Goldman SM, Korell M, et al. Rotenone, paraquat, and Parkinson's disease. Environ Health Perspect. 2011 Jun;119(6):866-72.

27 Martinez TN, Greenamyre JT. Toxin models of mitochondrial dysfunction in Parkinson's disease. Antioxid Redox Signal. 2012 May;16(9):920-34.

28 Wang C, Youssef J, Cunningham M, Badr M. Correlation between thyroid hormone status and hepatic hyperplasia and hypertrophy caused by the peroxisome proliferator-activated receptor alpha agonist Wy-14,643. J Carcinog. 2004 May;3(1):9.

29 Deng YT, Huang HC, Lin JK. Rotenone induces apoptosis in MCF-7 human breast cancer cell-mediated ROS through JNK and p38 signaling. Mol Carcinog. 2010 Feb;49(2):141-51.

30 Ding W, Xu C, Wang B, Zhang M. Rotenone Attenuates Renal Injury in Aldosterone-Infused Rats by Inhibiting Oxidative Stress, Mitochondrial Dysfunction, and Inflammasome Activation. Med Sci Monit. 2015 Oct;21: 3136-43.

31 Stallons LJ, Whitaker RM, Schnellmann RG. Suppressed mitochondrial biogenesis in folic acid-induced acute kidney injury and early fibrosis. Toxicol Lett. 2014 Jan;224(3):326-32.

32 Wallace DC. Mitochondrial diseases in man and mouse. Science. 1999 Mar;283(5407):1482-8.

33 Wallace DC. A mitochondrial paradigm of metabolic and degenerative diseases, aging, and cancer: a dawn for evolutionary medicine. Annu Rev Genet. 2005;39(1):359-407.

34 Bonet-Ponce L, Saez-Atienzar S, da Casa C, Sancho-Pelluz J, Barcia JM, Martinez-Gil N, et al. Rotenone Induces the Formation of 4-Hydroxynonenal Aggresomes. Role of ROS-Mediated Tubulin Hyperacetylation and Autophagic Flux Disruption. Mol Neurobiol. 2016 Nov;53(9):6194-208.

35 Li L, Dong X, Shu B, Wang Z, Hu Q, Zhong G. Iron-sulfur protein in mitochondrial complexes of Spodoptera litura as potential site for ROS generation. J Insect Physiol. 2014 Dec;71:21-9.

36 Zhou Q, Liu C, Liu W, Zhang H, Zhang R, Liu J, et al. Rotenone induction of hydrogen peroxide inhibits mTORmediated S6K1 and 4E-BP1/eIF4E pathways, leading to neuronal apoptosis. Toxicol Sci. 2015 Jan;143(1): 81-96.

37 Verma DK, Joshi N, Raju KS, Wahajuddin M, Singh RK, Singh S. Metabolic enhancer piracetam attenuates rotenone induced oxidative stress: a study in different rat brain regions. Acta Neurobiol Exp (Wars). 2015; 75(4):399-411.

38 Bengatta S, Arnould C, Letavernier E, Monge M, de Préneuf HM, Werb Z, et al. MMP9 and SCF protect from apoptosis in acute kidney injury. J Am Soc Nephrol. 2009 Apr;20(4):787-97.

39 Olson H, Betton G, Robinson D, Thomas K, Monro A, Kolaja G, et al. Concordance of the toxicity of pharmaceuticals in humans and in animals. Regul Toxicol Pharmacol. 2000 Aug;32(1):56-67.

40 Caboni P, Sherer TB, Zhang N, Taylor G, Na HM, Greenamyre JT, et al. Rotenone, deguelin, their metabolites, and the rat model of Parkinson's disease. Chem Res Toxicol. 2004 Nov;17(11):1540-8.

41 Zorov DB, Juhaszova M, Yaniv Y, Nuss HB, Wang S, Sollott SJ. Regulation and pharmacology of the mitochondrial permeability transition pore. Cardiovasc Res. 2009 Jul;83(2):213-25.

42 Kielar ML, John R, Bennett M, Richardson JA, Shelton JM, Chen L, et al. Maladaptive role of IL-6 in ischemic acute renal failure. J Am Soc Nephrol. 2005 Nov;16(11):3315-25.

43 Nechemia-Arbely Y, Barkan D, Pizov G, Shriki A, Rose-John S, Galun E, et al. IL-6/IL-6R axis plays a critical role in acute kidney injury. J Am Soc Nephrol. 2008 Jun;19(6):1106-15.

44 Li Y, Schwabe RF, DeVries-Seimon T, Yao PM, Gerbod-Giannone MC, Tall AR, et al. Free cholesterol-loaded macrophages are an abundant source of tumor necrosis factor-alpha and interleukin-6: model of NF-kappaB- and map kinase-dependent inflammation in advanced atherosclerosis. J Biol Chem. 2005 Jun;280(23):21763-72. 